\title{
SOME ASPECTS OF PARTICLE'S DYNAMICS
}

\author{
R.S.GOMES \\ Observatório Nacional Rio de Janeiro Brazil
}

October 18,1991

\begin{abstract}
.
Some features of the dynamics of particles affected by drag, in the field of the Sun and planets, are presented here. In particular mean motion and secular resonances are investigated. When dust particles are considered as a whole in the zodiacal cloud, a simple secular theory can explain much of its geometry. Dynamics of particles near an inner planet is mostly dispersive, but an average behavior can be deduced from some analytical and numerical considerations.
\end{abstract}

Key words: dust particles - drag - resonance

\section{Introduction}

Dust particle's dynamics is ruled by the combined effect of drag force (coming from radiation pressure) and the perturbation of the planets of the Solar System. Drag force alone accounts for the decay of the particle's semimajor axis and eccentricity. Other orbit elements are kept constant (mean longitude of course varies). Such a particle spirals towards the Sun with an increasingly circularized orbit.

When planets are included in dust particle dynamics, a lot of complications arise, due to the fact that, its semimajor axis decay maintained, the particle experiences a lot of dynamically relevant phenomena, including mean motion and secular resonances and passage by all inner planets.

The dynamics of a dust particle is highly dependent on the particle's size, density and other physical properties. These determine the radiation force imposed on the particle. The ratio of this force to the gravitational force is denoted by $\beta$. The decay rate for the semimajor axis and eccentricity as a function of $\beta$ can be found in (Wyatt and Whipple, 1950)

We divide this work in three parts, each one including a different aspect in a particle's dynamics, which are: mean motion resonances, secular variation of orbital elements (secular resonance) and passage near a planet.

\section{Mean Motion Resonances}

A dust particle coming towards the Sun by drag encounters many mean motion resonances on its course. Some are internal, meaning that the particle's orbit is inside that of the planet responsible for the resonance, and some are external. Dust particles are often trapped in external resonances, mainly with the Earth (Jackson and Zook, 1989). Trapping probability is associated with the particle's size, and its orbital elements just before resonance. Smaller particles, which are faster (towards the Sun), are more hardly trapped in resonance than larger ones. Trapping probability also increases with the disturbing planet's mass and depends on its orbital elements. Also more external planets have a greater probability to cause particles' trapping, because, for a given size, particles decay slower for higher distances to the Sun. 


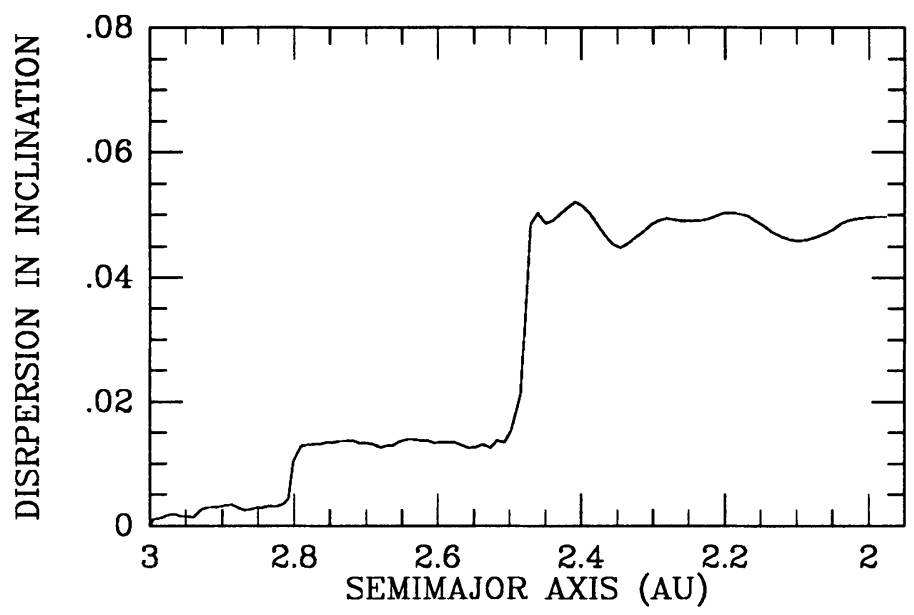

Fig. 1.

Dispersion in proper inclination of a set of 30 particles with $\beta=0.02$ and initial inclination $=10^{\circ}$. Particles are started with the same initial semimajor axis with inclination vectors ending on a circle whose center is the common forced inclination. The evolution of these points (ends of the inclination vectors) is tracked. At each output, a circle is fitted to those points. The square root of the sum of the squared distances of those points to the fitted circle divided by the number of points less 1 is the mentioned dispersion.

Any mean motion resonance causes a lot of dispersion in a particle's orbital elements. This dispersion can be defined by the experiment described next. We start numerical integrations with some particles, having initial equal semimajor axis, equal forced eccentricities and inclinations vectors (these depend only on the semimajor axis), equal proper eccentricities and inclinations, but different longitudes of pericenter and ascending nodes, in such a way that the eccentricity and inclination vectors have their ends distributed in circles. Also the initial longitudes of the particles are randomly selected. We can notice the evolution of that set of particles by watching the 'motion' of those starting circles. These circles, in fact, evolve quite smoothly, that is to say, the circles are maintained as near-circles for a long time, unless a dispersive factor is encountered. Mean motion resonances are one of these factors. The most dispersive internal resonance is the 3:1 resonance with Jupiter ( we consider particles with semimajor axis below $3 \mathrm{AU}$ ).

Figure 1 shows the dispersion in inclination experienced by a set of 30 particles with $\beta=0.02$, as they pass through several resonances with Jupiter. 


\section{Secular variation of Orbital elements}

To study the secular evolution of the orbital elements of small particles, we must put together the secular decay of the semimajor axis and eccentricity due to drag and the secular variations of other orbital elements due to planetary perturbations. So, in a first order, we have, for the secular decay of $a$ and $e$ due to drag:

$$
\begin{aligned}
& \left\langle\frac{\dot{a}}{a}\right\rangle=-\frac{2 \beta G M_{s}}{c a^{2}} \\
& \left\langle\frac{\dot{e}}{e}\right\rangle=-\frac{5}{2} \frac{\beta G M_{s}}{c a^{2}}
\end{aligned}
$$

where $G$ is the gravitation constant, $M_{s}$ the Sun's mass and $c$ the speed of light. Using complex numbers nomenclature, we define $z=e \exp i \varpi$ or $z=I \exp i \Omega$. The first order secular variation of these elements due to planetary perturbations, is given by:

$$
\dot{z}=i g z+i \sum \nu_{k} \exp i g_{k} t
$$

where $g$ is the proper frequency of the disturbed (massless) body, depending on its semimajor axis; $\nu_{k}$ is a coefficient also depending on the disturbed body's semimajor axis and related to the Solar System eigenfrequency $g_{k}$. When no drag is present (the classical approach for asteroids), the solution of $z$ is given by $\tilde{z}=\tilde{z}_{p}+\tilde{z}_{f}$, where:

$$
\begin{gathered}
\tilde{z}_{p}=z_{0} \exp i g t \\
\tilde{z}_{f}=-\sum \frac{\nu_{k}}{g-g_{k}} \exp i g_{k} t
\end{gathered}
$$

$\tilde{z}_{p}$ is called the proper component and $\tilde{z}_{f}$ the forced component.

This is a classical result that shows that the total $\tilde{z}$ is a sum of a vector, with constant modulus, rotating with the proper frequency $g$, and a sum of as many vectors as the Solar System eigenfrequencies considered, each rotating with that eigenfrequency.

When drag is present, the coefficients depending on the particle's semimajor axis can no longer be taken as constants in the above integrations, because they now have a secular decay. The solution of $z$ for particles affected by drag is given by:

$$
\begin{gathered}
z_{p}=z_{0} \exp i G(t) \\
z_{f}=z_{p} \int z_{p}^{-1} F d t \\
\text { where } \quad F=i \sum \nu_{k} \exp i g_{k} t
\end{gathered}
$$

The decomposition of $z$ into a proper and a forced element, as shown above, is somewhat unnatural for particles. In fact $z_{p}$ rotates with the particle's proper frequency $g$, which varies with the particle's semimajor axis. $G(t)$ is an integral of $g[a(t)]$. On the other hand, we can no longer say that $z_{f}$ is a sum of vectors rotating with the Solar System eigenfrequencies. A good way to study the behavior of $z_{f}$ when drag is present is to consider variables $W$ and $Y$, defined by

$$
W=z_{f} \exp -i g_{k} t
$$



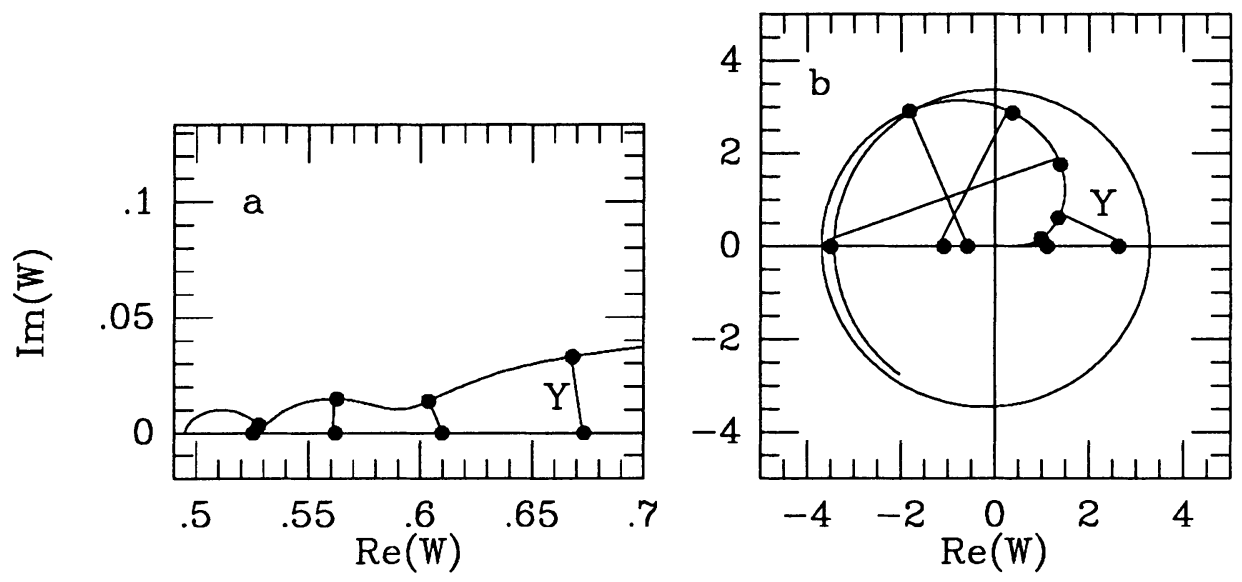

Fig. 2.

Figure $2 a$ is an amplification of the beginning of the variation in figure 2b. In each of both graphs, we see 3 curves: $W, Y$ and $W-Y$, which is the static component of the forced element in a frame rotating with the corresponding Solar System eigenfrequency. On this frame $W-Y$ remains on the $X$-axis. $W$ is the dynamical forced element (component) on the same frame. In both graphs it is shown as a curve evolving. $Y$ is shown as a set of vectors starting on the $X$-axis $(W-Y)$ and ending on the curve $W$.

$$
Y=\left(z_{f}-\tilde{z}_{f}\right) \exp -i g_{k} t
$$

The equations for $W$ and $Y$ are

$$
\begin{aligned}
& \dot{W}=i\left(g-g_{k}\right) W-i \nu \\
& \dot{Y}=i\left(g-g_{k}\right) Y-\dot{M}_{k}
\end{aligned}
$$

where $M_{k}=\frac{\nu_{k}}{g-g_{k}}$ Figures $2 \mathrm{a}$ and $2 \mathrm{~b}$ show the variation of $W$ and $Y$, for the inclination case, with only Jupiter and Saturn as disturbing planets. $Y$ is shown as vectors with origin in the $\mathrm{X}$-axis and end on the curve $W$. Figure 2a shows the details in the beginning of the variation and figure $2 \mathrm{~b}$ shows all the evolution to near the Sun, passing through secular resonance. For $t=0, z_{f}=\tilde{z}_{f}$ and $Y=0$. If $M_{k}$ were constant (non-drag case) $Y$ would be constantly equal to zero as expected. In the drag case, $\dot{M}_{k}$ creates an instant increase of $|Y|$ on the X-axis. Then $i\left(g-g_{k}\right)$ makes $Y$ rotate. $Y$ also gives an idea of how the drag forced element gets far from the non-drag forced element. Figure $2 \mathrm{~b}$ shows the point where secular resonance is encountered, that is when $W$ has a tangent parallel to the $\mathrm{Y}$-axis, at a point not on the $\mathrm{X}$-axis. Long past the secular resonance, $Y$ essentially rotates with frequency equal to $g-g_{k}$ centered at a slowly varying point on the negative half of the $\mathrm{X}$-axis. In a sense, one can see the equation for $Y$ as one giving rise to the creation of 
instantly new proper element. If, at some point, drag ceased to affect the particle, it would have its forced component associated to its semimajor axis (as in the nondrag case) and a proper element added by $Y$ at the point where drag ceased to exist and then evolve according to the classical non-drag approach.

\section{Passage by a Planet}

Equation 1 is really deduced from a more basic expression involving the planet's orbital elements, given below (inclination case):

$$
\dot{z}=i g z-i \Sigma A_{j} z_{j}
$$

where the $A_{j} s$ depend on Laplace coefficients and $z_{j}$ are the planets' (secular) inclination vectors, which developed in terms of the Solar System eigenmodes, give rise to equation 1 . On the other hand, we have $g=\sum A_{j}$ (inclination case), which allows us to write

$$
\left(z-z_{k}\right)=i\left[\Sigma A_{j}\left(z-z_{j}\right)\right]-\dot{z_{k}}
$$

$A_{k}$ is particularly high in the neighborhood of planet $k$, which yields the approximate expression, for a neighborhood of a planet:

$$
\dot{\zeta_{k}} \cong i A_{k} \zeta_{k}
$$

where $\zeta_{k}=z-z_{k}$ is the inclination vector measured from planet k's orbital plane. This shows us that in the neighborhood of a planet, the inclination vector of a particle essentially rotates around that of the nearby planet. This is also an approximate result due to the fact that the real motion in the proximity of a planet is very dispersive and the effective rate of variation of the node highly depends on the value of the inclination.

Figures $3 \mathrm{a}$ and $3 \mathrm{~b}$ help us to understand the dynamics of particles crossing a region gravitationally dominated by an inner planet. We integrated around 1000 particles in the field of the Sun and Earth alone, each particle started at $1.15 \mathrm{AU}$ and coming as far as $0.85 \mathrm{AU}$. They were started with randomly chosen initial inclinations from 0 to 10 degrees, and an initial eccentricity equal to 0.05 . All particles associated to $\beta=0.12$. At $0.85 \mathrm{AU}$ we measure each particle's inclination and longitude of the node and take the differences from the initial conditions. Figure 3a shows $\Delta \Omega$ against initial inclination. Each point represents the result of a particular particle. One notices the expected tendency in the decrease of $\Delta \Omega$ with the increase of initial inclination. Also the dispersion is smaller.

Figure $3 \mathrm{~b}$ may be more elucidative as we assume that the particles analized in our simulation are started with their inclination vectors on a circle, then we sum to each initial inclination vector on the circle their $\Delta \Omega$ and $\Delta I$,coming from the numerical integration above mentioned. The final inclinations are shown as points spread out in an irregular manner.

\section{Some Practical Conclusions}

The notion of forced dynamical elements for dust particles is a basic concept in defining symmetry planes for the zodiacal cloud. In particular, inclination vectors 

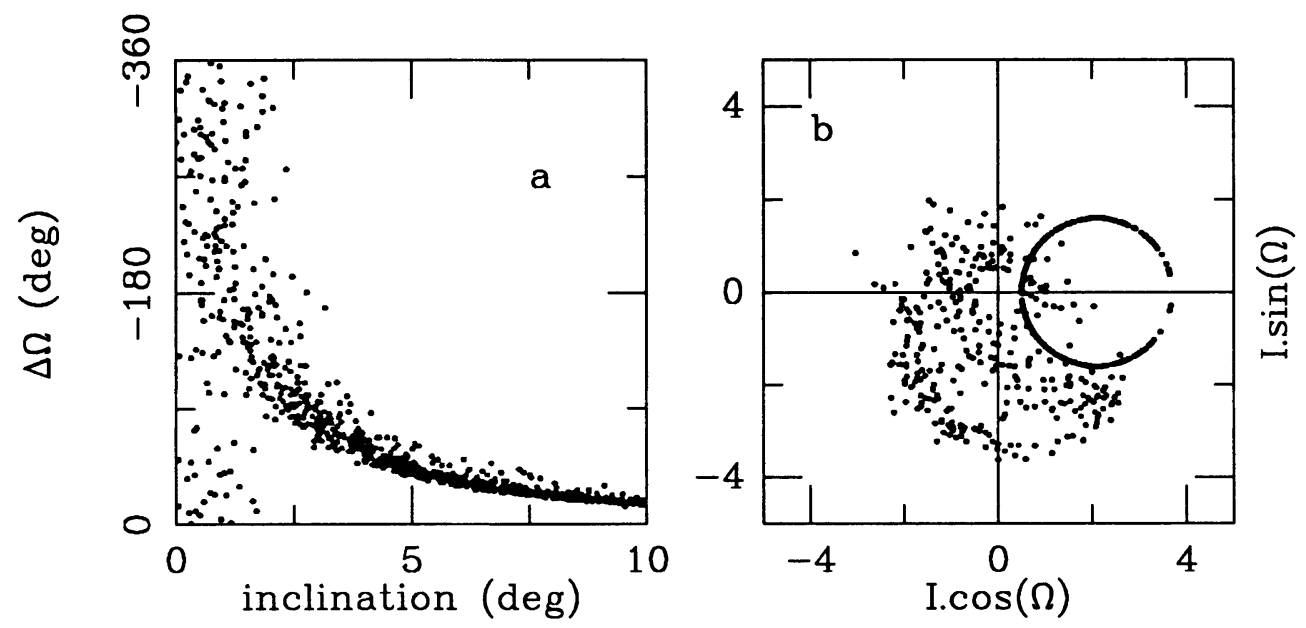

Fig. 3.

In a, the variation of the inclination vector near a planet is mostly a rotation of that vector around the inclination vector (end) of that planet, with increasingly angular frequency, as closer to the planet the particle gets. In b, particles affected by drag, started near the Earth, with inclination vectors (ends) distributed on a circle evolve in such a way that the circle is distorted due to different angular frequencies for particles with different inclinations (figure $3 a$ ).

with ends located on circles are related to the observation of bands, which have been associated to the Hyrayama family of asteroids ( Dermott et al, 1984). The evolution of these circles as particles spiral towards the Sun reflects the evolution of such bands. The maintenance of an approximate circle reflects the surviving of the bands to regions closer to the Sun. The center of that approximate circle can be associated to the symmetry plane of the bands. Near an inner planet, the circle method breaks down, because of dispersion and different angular frequencies for the node. A symmetry plane is hard to define but it tends to the planet's orbital plane as the size of the particle is bigger. Of course all the dynamics of particles depend on its size, in particular the variation of the symmetry plane in respect with the particle's mean distance to the Sun. Passage by mean motion resonances with Jupiter tends to disperse the orbital elements but with no great harm to the method described above. On the other hand, when particles are trapped in resonance with an inner planet that contributes to positioning the symmetry plane to near the orbital plane of that planet.

\section{References}

Dermott,S.F.;Nicholson,P.D.;Burns,J.A.;Houck,J.R. 1984 Nature 312, 505-509

Jackson,A.A.; Zook,H.A. 1989 Nature 337, 629

Wyatt,S.P.; Whipple,F.L. 1950 A strophys. J. 111, 134-141 